\title{
technische universität
} dortmund

\author{
Investition und Finanzierung
}

\section{Optimales Anlagevermögen von Versicherungsunternehmen}

Matthias Pelster

Florian Kaposty

Working Paper Nr. 1/2014 


\section{Ansprechpartner:}

Dr. Matthias Pelster, e-mail: matthias.pelster@tu-dortmund.de

Die Arbeitspapiere Investition und Finanzierung erscheinen in loser Folge. Mit ihnen werden Aufsätze (oft als Preprint), sowie Projektberichte und Vorträge publiziert. Die Arbeitspapiere sind daher nicht unbedingt endgültig abgeschlossene wissenschaftliche Beiträge. Die Reihe hat das Ziel, der Fachöffentlichkeit Arbeiten aus dem Bereich der Finanzwirtschaft der Wirtschafts- und Sozialwissenschaftlichen Fakultät der Technischen Universität Dortmund vorzustellen. Anregungen und kritische Kommentare sind nicht nur willkommen, sondern ausdrücklich erwünscht. 


\title{
Optimales Anlagevermögen von Versicherungsunternehmen
}

\author{
Matthias Pelster; Florian Kaposty*
}

13. Mai 2014

\begin{abstract}
Versicherungsunternehmen (VU) unterliegen detaillierten regulatorischen Vorschriften und werden in besonderem Maße behördlich beaufsichtigt. Gegenwärtig erfolgt die Einführung einheitlicher europäischer Regulierungsvorschriften im Rahmen des Projekts Solvency II, wodurch die aufsichtsrechtlichen Anforderungen an VU im EU-weiten Binnenversicherungsmarkt harmonisiert werden sollen. Ziel des Beitrags ist die Bestimmung des optimalen Anlagevermögens eines im Marktumfeld von Solvency II agierenden VU.
\end{abstract}

Insurers are subject to elaborate regulation. Currently the EU implements the Solvency II Directive that codifies and harmonizes the EU insurance regulation. Our contribution analyzes the optimal invested capital of an insurance company subject to the Solvency II Directive.

Keywords: Risikomanagement, Solvency II, Value at Risk, Terminabsicherung, Anlageentscheidung.

JEL Classification Numbers: G00, G22.

\footnotetext{
*Correspondence: Matthias Pelster, University TU Dortmund, 44221 Dortmund, Germany. Tel. +49-231-755-3788. E-mail: matthias.pelster@tu-dortmund.de
} 


\section{Regulierung von Versicherern}

Versicherungsunternehmen (VU) besitzen eine exponierte Stellung in der europäischen Wirtschaft und sind wichtige Akteure auf den internationalen Finanzmärkten. Aus diesen Gründen unterliegen Versicherer detaillierten regulatorischen Vorschriften und werden in besonderem Maße behördlich beaufsichtigt. Wichtiger Bestandteil eines funktionierenden Regelwerks ist die kontinuierliche Anpassung bestehender Vorschriften an aktuelle gesellschaftliche, politische und wirtschaftliche Entwicklungen. Daher erfolgt die Einführung einheitlicher europäischer Regulierungsvorschriften im Rahmen des Projekts Solvency II, wodurch die aufsichtsrechtlichen Anforderungen an Versicherungsunternehmen im EU-weiten Binnenversicherungsmarkt harmonisiert werden sollen (Vgl. Schradin, 2003, S. 36f.). Neben den mit der Einführung und fortdauernden Weiterentwicklung von Solvency II einhergehenden Herausforderungen werden die europäischen Versicherer durch die konjunkturelle und wirtschaftliche Entwicklung der vergangenen Jahre außerordentlich beeinflusst.

VU betreiben sowohl zur Erwirtschaftung des zur Erfüllung vertraglicher Zinsverpflichtungen gegenüber den Versicherungsnehmern notwendigen Kapitals als auch unter Renditeaspekten ein ausgeprägtes Kapitalanlagegeschäft, weshalb die wirtschaftlich angespannte Situation eine besondere Fokussierung auf die Kapitalmarktaktivität der Versicherer verlangt. Insbesondere die in Folge der Finanzkrise von 2007 seitens der Europäischen Zentralbank (EZB) gewollte und weiterhin anhaltende Niedrigzinspolitik führt zu einer signifikanten Belastung der europäischen Versicherer. Nach aktuellen Schätzungen führte die Niedrigzinsphase im Jahr 2012 zu zinsbedingten Mindereinnahmen für die Versicherer von annährend vier Milliarden Euro (Vgl. Gesamtverband der Deutschen Versicherungswirtschaft e.V., 2013, S. 6). Die Niedrigzinsphase trifft insbesondere die Lebensversicherer, da diese unter einem erheblichen Renditedruck stehen, um die Erwirtschaftung des vertraglich fixierten Garantiezinses sicherstellen zu können. Ursächlich hierfür ist, dass der durchschnittliche Garantiezins der deutschen Lebensversicherer bei etwas über $3 \%$ p.a. liegt und somit deutlich über dem aktuell gültigen Leitzins der EZB von 0,5\% p.a. (Assekurata GmbH, 2013, S. 12). Aus diesem Grund strukturieren einige deutsche Lebensversicherer ihre Produkte um und verzichten auf die Gewährung des Garantiezinses.

Der Anlagepolitik von Versicherern kommt somit eine wachsende Bedeutung zuteil. VU haben bei Festlegung ihrer Anlageentscheidung einerseits eine ausreichende Solvabilität gemäß den aufsichtsrechtlichen Regelungen von Solvency II und andererseits bestehende Zinsverpflichtungen an die Versicherungsnehmer sowie bilanzielle Anforderungen zu berücksichtigen. 


\section{Modell eines kompetitiven VUs}

Die verstärkte Kapitalmarktorientierung seitens der Versicherer und eine damit einhergehende vermehrte Aktivität auf Kapitalmärkten impliziert die Übernahme neuer Risikoquellen, welche den Marktwert des Versicherers beeinflussen. Ein funktionierendes Risikomanagement ist somit für VU zur Steuerung der eingegangenen Risiken von hoher Relevanz. Durch veränderte geschäftspolitische Rahmenbedingungen, die sowohl durch die neuen aufsichtsrechtlichen Vorschriften von Solvency II als auch aufgrund eines erhöhten Wettbewerbsdrucks und volatiler Kapitalmärkte entstanden sind, hat die Bedeutung eines adäquaten Risikomanagements in den letzten Jahren stark zugenommen. Versicherer haben folglich darüber zu entscheiden, in welchem Umfang risikobehaftete Anlagen im Aktivgeschäft gehalten werden sollen.

Weitere relevante Zielgrößen der Unternehmenssteuerung sind im Passivgeschäft das Volumen der Rückstellungen und der Umfang der vorhandenen Eigenmittel. Letztere Größe ist dabei invariabel mit den Solvabilitätsvorschriften von Solvency II verknüpft, welche eine angemessene Eigenmittelbasis zur Sicherung der fortlaufenden Unternehmenstätigkeit und zum Schutz der Versicherungsnehmer fordern (Vgl. Farny, 2011, S. 11; Nguyen, 2008, S. 23; Wiesemann, 2011).

Grundlage der weiteren Analysen ist ein Modell eines kompetitiven, risikoaversen VU. Der Versicherer ist aufgrund unsicherer Renditen in den gehaltenen Anlagen einem Gewinnrisiko ausgesetzt. Hierdurch kann die Solvenz des Unternehmens gefährdet werden, sodass seitens des Managements über die optimale Handhabung der Risiken $\mathrm{zu}$ entscheiden ist.

Im Folgenden wird ein Zwei-Zeitpunkte-Modell mit den Zeitpunkten $t_{0}$ und $t_{1}$ betrachtet. Das VU hat alle Entscheidungen zu Beginn der Planungsperiode zu treffen. Ausgangspunkt zur Festlegung der optimalen Geschäftspolitik ist die in Abbildung 1 dargestellte, auf einer marktnahen Bewertung der Bilanzposten basierende Solvenzbilanz eines Versicherungsunternehmens.

\begin{tabular}{l|l} 
Aktiva & \multicolumn{1}{l}{ Passiva } \\
\hline Anlagen $A$ & $\begin{array}{l}\text { Rückstellungen } R \\
\text { Eigenmittel } E\end{array}$
\end{tabular}

Abbildung 1: Solvenzbilanz eines Versicherungsunternehmens.

Die Passivseite der Versicherungsbilanz ist durch die Rückstellungen als größtem Passivposten gekennzeichnet. Ursächlich hierfür ist, dass die Ausübung der klassischen Versicherungstätigkeit die Bildung von Rückstellungen zur Begleichung zukünftiger, 
potentieller Schäden bedingt (vgl. Doff, 2007, S. 12). Die Rückstellungen sind abhängig vom fest vorgegebenen Versicherungsumfang $V$ und somit bereits fixiert. Es gilt $R=R(V)$. Zusätzlich hält das Unternehmen Eigenmittel zur Absicherung unerwarteter Verluste. Im Aktivgeschäft investiert das Versicherungsunternehmen in risikobehaftete Anlagen und hält ein Anlagenportfolio zum Marktwert $A$. Wesentliches Ziel der Kapitalanlage ist im Allgemeinen nicht der Eigenhandel, sondern die Erwirtschaftung des zur Absicherung der Erfüllbarkeit der bestehenden versicherungstechnischen Verpflichtungen notwendigen Kapitals. Exemplarisch sei die Erwirtschaftung des vertraglich fixierten Garantiezinses in der Lebensversicherung genannt. Eine Verfehlung dieser Mindestrenditeziele führt zum Verlust der Wettbewerbsfähigkeit des Versicherers und somit langfristig zu dessen Insolvenz. Die zum Zeitpunkt $t_{0}$ der Anlageentscheidung unsichere Rendite des Anlagenportfolios $A$ werde mit $r_{A}$ bezeichnet.

Die Anlagenrendite sei normalverteilt mit Erwartungswert $\mu_{A}$ und Varianz $\sigma_{A}^{2}$, d.h. es gilt $\tilde{r}_{\mathrm{A}} \sim \mathcal{N}\left(\mu_{A}, \sigma_{A}^{2}\right)$. Die Normalverteilungsannahme entspricht den theoretischen Anforderungen zur Berechnung der Solvenzkapitalanforderungen (SCR) unter Solvency II (vgl. Committee of European Insurance and Occupational Pensions Supervisors, 2007). Die Finanzierung der Anlagen erfolgt aus den vorhandenen Eigenmitteln, Teilen der Rückstellungen sowie aus den nicht zur sofortigen Schadenregulierung benötigten, vereinnahmten Prämien (vgl. Albrecht, 1995).

Bei Festlegung des optimalen Anlagevolumens $A$ in $t_{0}$ hat das Versicherungsunternehmen die Bilanzgleichung

$$
A=E+R
$$

zu erfüllen.

Dem Versicherungsunternehmen entstehen durch die Anlagetätigkeit operative Kosten $C(A)$, die vom Bilanzvolumen $A$ und somit auch vom gewählten Anlagevolumen abhängen. Die Kosten der Anlagetätigkeit werden durch die auf das Periodenende $t_{1}$ bezogene, quadratische Kostenfunktion

$$
C(A):=\frac{1}{2} \theta A^{2}, \quad \theta>0
$$

abgebildet. Das Unternehmen besitzt positive und steigende Grenzkosten. Ferner hat das VU Zahlungsverpflichtungen aus den Rückstellungen an die Versicherungsnehmer am Periodenende zu leisten. Die Höhe der in $t_{1}$ zu entrichtenden Zahlungen ist durch den deterministischen Zinssatz $r_{\mathrm{R}}$ in $t_{0}$ festgelegt. Dieser entspricht den durchschnittlichen Zinsverpflichtungen aus den gesamten Rückstellungen. Zinsverpflichtungen aus Rückstellungen gegenüber den VN entstehen dem Versicherer 
etwa in Form der Deckungsrückstellung in der Lebensversicherung oder der Rentendeckungsrückstellung in der Haftpflichtversicherung (vgl. Wagner, 2001). Da dem VU zum Planungszeitpunkt $t_{0}$ der gegenwärtige Vertragsbestand und somit auch die vertraglich fixierten Zahlungen aus den Rückstellungen bekannt sind, ist die Annahme deterministischer Zinsverpflichtungen begründet.

Neben dem bereits beschriebenen Kapitalanlagegeschäft betreibt ein VU als Kerntätigkeit das klassische Versicherungsgeschäft. Aus der Versicherungstätigkeit erzielt das VU unter Berücksichtigung der ihm entstehenden Kosten ein Ergebnis in Höhe von $G$, welches vom Volumen der Versicherungstätigkeit $V$ abhängt, es gilt $G=G(V)$. Das Ergebnis aus dem Versicherungsgeschäft wird als deterministisch angenommen um den Fokus nicht von der Bestimmung des optimalen Anlagevermögens eines VU zu verrücken. Des Weiteren wird angenommen, dass das VU keine Rückversicherung nutzt, wodurch die SCR mindernde Effekte durch einen Risikotransfer auf den Rückversicherer ausgeschlossen werden. Zur Vernachlässigung von Zinseffekten wird ein risikoloser Zinssatz von $0 \%$ pro Periode unterstellt.

Zusammenfassend kann der risikobehaftete Gewinn $\tilde{\Pi}$ des VUs am Periodenende $t_{1}$ angegeben werden:

$$
\tilde{\Pi}=\tilde{r}_{A} A-r_{R} R-C(A)+G(V) .
$$

Aufgrund der unsicheren Erträge in den Anlagen ist das VU einem Endvermögensrisiko ausgesetzt. Hierdurch kann die Solvabilität gefährdet werden. Zur Bestimmung des optimalen Anlagevermögens ist eine durch Solvency II geforderte ausreichende Solvenzkapitalausstattung zu beachten, um das Risiko hoher Verluste adäquat absichern zu können. Solvency II stellt mit dem Value at Risk ein RisikomanagementWerkzeug bereit, mit dem das Endvermögensrisiko gesteuert werden kann.

\section{Berücksichtigung von Solvency II und Value at Risk als Nebenbedingungen}

Die Solvenzpolitik des Versicherers ist derart zu gestalten, dass die aus den Rückstellungen resultierenden Zahlungsverpflichtungen fristgerecht und vollständig mit hoher Wahrscheinlichkeit bedient werden können. Kann der Versicherer diesen Verpflichtungen nicht nachkommen, tritt Insolvenz ein. Vereinfacht ausgedrückt schreibt Solvency II vor, dass mindestens Eigenmittel in Höhe der SCR vorzuhalten sind. Somit hat das VU die Bedingung 


$$
E>\mathrm{SCR}
$$

zu erfüllen und diese bei der Bestimmung der optimalen Anlagepolitik zu berücksichtigen. Das VU orientiert sich bei der Bestimmung des benötigten Solvenzkapitals an der Standardformel von Solvency II. Der Value at Risk wird in die Solvenzpolitik integriert und zur Steuerung der Insolvenzwahrscheinlichkeit verwendet (zum Value at Risk vgl. z.B. Jorion, 2000). Seitens Solvency II ist festgelegt, dass die SCR dem Value at Risk zum Konfidenzniveau $\alpha=0,995$, kalibriert über eine Periode zu entsprechen haben, d.h. es gilt

$$
\mathrm{SCR}=\mathrm{VaR}_{0,995}
$$

Gleichung (5) ermöglicht die Bestimmung des benötigten Solvenzkapitals mittels des Value at Risks, was im Folgenden weiter ausgeführt wird. Abbildung 2 veranschaulicht das Zusammenspiel von SCR und VaR.

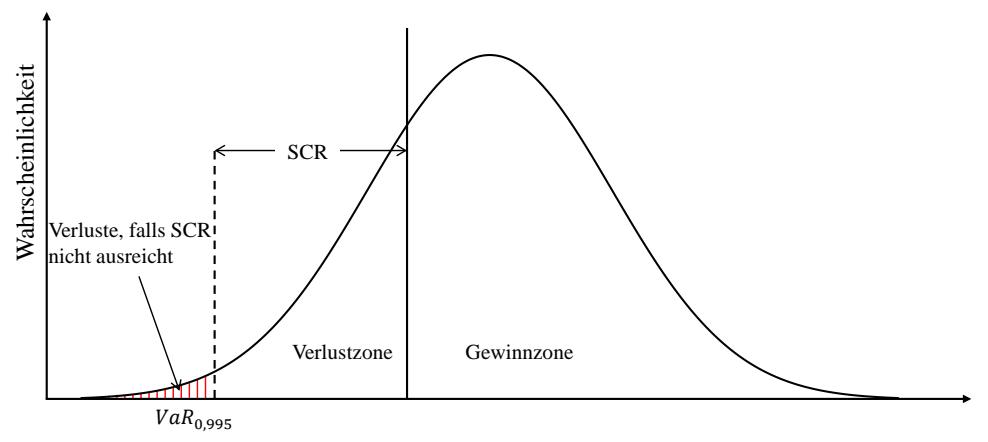

Abbildung 2: Gewinn und Verlust bezogen auf die Solvenzbilanz

Die Solvabilität und damit auch der VaR werden durch die stochastische Anlagenrendite beeinflusst. Der Value at Risk des Anlagenrisikos operationalisiert die Zahlungsverpflichtungen des Versicherers, indem das Aktiv- und Passivgeschäft des VUs mit dessen Eigenmittelausstattung in Relation gesetzt wird. Ausgangspunkt hierfür ist die durch das Unternehmen zu erfüllende Solvenzbedingung, welche für die weiteren Analysen wie folgt festgelegt wird:

Die Summe aus dem Marktwert der Anlagen am Periodenende und dem operativen Ergebnis aus dem klassischen Versicherungsgeschäft darf höchstens mit Wahrscheinlichkeit $1-\alpha$ kleiner als die aus den Rückstellungen zu leistenden Zahlungsverpflichtungen sein. Formal gilt: 


$$
\mathbf{P}\left[\left(1+\tilde{r}_{\mathrm{A}}\right) A+G(V)-r_{\mathrm{R}} R<0\right]=1-\alpha .
$$

Um den bereits angesprochenen Zusammenhang zwischen Aktiv- und Passivgeschäft und Eigenmittelausstattung herzustellen, wird die Solvenzbedingung unter Beachtung der Bilanzgleichung (1) äquivalent umgeformt zu

$$
\mathbf{P}\left[-\tilde{r} A>r_{\mathrm{R}} E+G(V)\right]=1-\alpha .
$$

Aufgrund der normalverteilten Anlagenrendite folgt $\tilde{r}:=1+\tilde{r}_{\mathrm{A}}-r_{\mathrm{R}}$ einer Normalverteilung mit Erwartungswert $\mu=\mathbf{E}[\tilde{r}]=1+\mu_{A}-r_{\mathrm{R}}$ und Varianz $\sigma^{2}=\operatorname{Var}[\tilde{r}]=\sigma_{A}^{2}$. Standardisieren von $\tilde{r}$ zu einer standardnormalverteilten Zufallsvariable ermöglicht die äquivalente Formulierung der Solvenzbedingung als

$$
E=\frac{-\left(\mu+\sigma \Phi^{-1}(1-\alpha)\right) A-G(V)}{r_{\mathrm{R}}}
$$

wobei $\Phi^{-1}(1-\alpha)$ das $(1-\alpha)$-Quantil der Standardnormalverteilung bezeichnet.

Durch Gleichung (6) ist eine vorläufige Charakterisierung der vorzuhaltenden Eigenmittel gegeben. Bestandteil dieser Gleichung wie auch der Gewinngleichung (3) ist das Ergebnis des operativen Versicherungsgeschäfts $G=G(V)$. Diese Größe ist für die weiteren Analysen mit der Bilanzgleichung (1) in Verbindung zu bringen.

Sowohl das Ergebnis des klassischen Versicherungsgeschäfts als auch die gebildeten Rückstellungen hängen vom zum Entscheidungszeitpunkt $t_{0}$ bereits fixierten Versicherungsvolumen $V$ ab, wodurch eine Verbindung beider Größen gegeben ist. Da das VU annahmegemäß bereits in $t_{0}$ das Ergebnis der reinen Versicherungstätigkeit in Abhängigkeit des Versicherungsvolumens kennt, ist es dem Unternehmen aufgrund der vereinfachten Modellstruktur möglich, auf das eingesetzte Versicherungsvolumen zu schließen. Weil weiterhin wegen $R=R(V)$ auch die Höhe der Rückstellungen durch das Versicherungsvolumen bestimmt ist, kann das VU über das Versicherungsvolumen einen Zusammenhang zwischen dem Ergebnis des reinen Versicherungsgeschäfts und den Rückstellungen herstellen.

Für die weitere Modellierung wird ein linearer Zusammenhang zwischen $R(V)$ und $G(V)$ angenommen:

$$
G(V):=\beta R(V), \quad \beta \in \mathbb{R}
$$


Es folgt

$$
E=\underbrace{\frac{-\left(\mu+\sigma \Phi^{-1}(1-\alpha)+\beta\right)}{r_{\mathrm{R}}-\beta}}_{=: c_{\alpha}} A,
$$

d. h. die Höhe der mindestens vorzuhaltenden Eigenmittel ist gegeben durch

$$
E=c_{\alpha} A
$$

Durch das VaR-Konzept wurden die in den Gleichungen (4) und (5) formulierten Solvenzkapitalanforderungen zu obiger Bedingung (9) verknüpft. Die in der Eigenmittelbedingung (9) enthaltenen Parameter haben $c_{\alpha} \in(0,1)$ zu erfüllen. Hierdurch werden die zur SCR-Bedeckung vorzuhaltenden Eigenmittel in einen direkten Zusammenhang mit den gehaltenen Anlagen gesetzt. Hält das Versicherungsunternehmen Eigenmittel in der durch Gleichung (9) bestimmten Höhe vor, so ist dessen Solvenz entsprechend den Vorgaben von Solvency II mit einer Wahrscheinlichkeit von 99, 5 \% gesichert.

Zur Festlegung der optimalen Anlagepolitik ist unter Berücksichtigung der Risikoaversion des betrachteten VU eine Entscheidungsregel zu formulieren. Der Versicherer wählt dasjenige Anlagevolumen, welches den erwarteten Nutzen des Gewinns maximiert. Dabei wird die Risikoeinstellung des Versicherers durch eine zweimal stetig differenzierbare, beschränkte von Neumann-Morgenstern-Nutzenfunktion $U(\tilde{\Pi})$ mit positivem, fallendem Grenznutzen abgebildet (vgl. von Neumann und Morgenstern, 2007).

Es ergibt sich das Maximierungsproblem

$$
\begin{array}{ll}
\max _{A} & \mathbf{E}[U(\tilde{\Pi})], \\
\text { sodass } & A=E+R(V), \\
& G(V)=\beta R(V), \\
& E=c_{\alpha} A .
\end{array}
$$

Einbinden der Nebenbedingungen in die Gewinngleichung ergibt

$$
\tilde{\Pi}=A\left(\tilde{r}_{\mathrm{A}}+\left(\beta-r_{\mathrm{R}}\right)\left(1-c_{\alpha}\right)\right)-\frac{1}{2} \theta A^{2} .
$$

Die Zielfunktion $\mathbf{E}[U(\tilde{\Pi})]$ ist streng konkav, da die Nutzenfunktion $U(\tilde{\Pi})$ als streng konkav vorausgesetzt wurde und der Gewinn eine im Anlagevolumen $A$ streng konkave Funktion ist. Daher ist die Bedingung erster Ordnung notwendig und hinreichend 
zugleich für ein globales, eindeutiges Maximum der Zielfunktion. Das optimale Anlagevolumen $A^{*}$ ergibt sich aus der Bedingung erster Ordnung: ${ }^{1}$

$$
\mathbf{E}\left[U^{\prime}\left(\tilde{\Pi}^{*}\right) \tilde{r}_{\mathrm{A}}\right]+\left(\beta-r_{\mathrm{R}}\right)\left(1-c_{\alpha}\right) \mathbf{E}\left[U^{\prime}\left(\tilde{\Pi}^{*}\right)\right]-\theta A^{*} \mathbf{E}\left[U^{\prime}\left(\tilde{\Pi}^{*}\right)\right]=0 .
$$

Satz 3.1. (Optimale Anlagepolitik)

Für die optimale Anlagepolitik des VU gilt:

1.Der erwartete Nutzen des Gewinns wird maximiert, wenn der Versicherer das durch Gleichung (15) gegebene Anlagevolumen realisiert. Die optimale Anlagepolitik wird sowohl durch Marktfaktoren als auch durch seitens des Unternehmens festgelegte Größen beeinflusst. Zu den Marktfaktoren zählen die Grenzkosten und zu den unternehmensspezifischen Einflussfaktoren der Grad der Risikoaversion, der Proportionalitätsfaktor zwischen Rückstellungen und Ergebnis des klassischen Versicherungsgeschäfts sowie die Erwartungen hinsichtlich der stochastischen Anlagenrendite. Ferner beeinflusst das seitens der Aufsichtsbehörde festgelegte Solvenzniveau die optimale Anlagepolitik.

2.Durch Verwendung des Value at Risk-Konzepts zur Bestimmung der optimalen Entscheidungspolitik kann ausgehend vom Anlagevolumen über die Höhe der vorzuhaltenden Eigenmittel nicht getrennt entschieden werden. Der Umfang von Aktiv- und Passivgeschäft ist durch das VU simultan festzusetzen.

Das im vorliegenden Modell von dem Versicherer verlangte simultane Aktiv- und Passivmanagement zeigt insbesondere, dass die Höhe der zur Bedeckung der Solvenzkapitalanforderungen vorzuhaltenden Eigenmittel nicht direkt anhand der VaRNebenbedingung (5) bestimmt werden können. Vielmehr sind die Eigenmittel unter Berücksichtigung des gesamten Modellrahmens ausgehend von der optimalen Anlageentscheidung zu kalkulieren.

Es wird angemerkt, dass der Einfluss einer Erhöhung der Solvenzwahrscheinlichkeit auf die optimale Anlagepolitik unbestimmt ist. Dies verdeutlicht die komplexen Abhängigkeitsstrukturen zwischen dem Anlagevolumen und den in Satz 3.1 genannten Determinanten der optimalen Anlagepolitik.

Die zu Periodenbeginn realisierten Entscheidungen werden in diesem Kapitel vom VU vor dem Hintergrund einer fehlenden Möglichkeit des Risikotransfers getroffen. Bei Festlegung der optimalen Entscheidungspolitik kann das mit der stochastischen Anlagenrendite einhergehende Endvermögensrisiko annahmegemäß nicht auf dem Terminmarkt abgesichert werden. In der Praxis sind VU jedoch wichtige Akteure

\footnotetext{
${ }^{1}$ Ein * kennzeichnet optimale Größen.
} 
auf den Finanzmärkten und nutzen diese nicht nur unter Renditeaspekten, sondern auch zur Risikoabsicherung.

\section{Terminkontrakte}

Ein funktionierendes Risikomanagement ist für Versicherer von existentieller Bedeutung. Einen wesentlichen Beitrag liefert die Möglichkeit der Risikoabsicherung mittels Derivaten. Sie bieten dem VU die Möglichkeit, Risiken auf einem Risikomarkt zu verkaufen und so potentielle Gewinnschwankungen zu mindern. Dies erfolgt durch den Einsatz passender Finanzinstrumente im Rahmen des sogenannten Hedgings.

Im Rahmen dieses Beitrags nutzt das VU fortan Forwards auf einem kompetitiven Terminmarkt zur Absicherung der risikobehafteten Anlagenrendite. Der Terminkurs sei durch $r_{f}$ gegeben. Das Kontraktvolumen der Terminabsicherung, $H$, ist zum Planungszeitpunkt $t_{0}$ neben dem Anlagevolumen festzulegen. $H>0$ bezeichnet einen Verkauf per Termin. Die Laufzeit der Forwards stimme mit dem Planungszeitraum des Versicherers überein.

Um die preisausgleichende Wirkung in den Gewinnschwankungen durch das entgegengerichtete Termingeschäft abzubilden, wird eine perfekt negative Korrelation zwischen dem Gewinn aus der Anlagetätigkeit, $\tilde{r}_{\mathrm{A}} A$, und dem Ergebnis des Forwardgeschäfts, $\left(r_{f}-\tilde{r}_{\mathrm{A}}\right) H$, angenommen. Hieraus folgt nicht direkt eine Vollabsicherung der Risikoposition, da die Diversifikation das Verhältnis von vertraglich fixiertem Terminkurs zu erwarteter zukünftiger Anlagenrendite berücksichtigt.

Es wird davon ausgegangen, dass das Forward-Hedging den SCR nicht beeinflusst. Der Einsatz von Risikominderungstechniken kann unter Solvency II das vorzuhaltende Solvenzkapital reduzieren, wobei der genaue Einfluss auf den SCR individuell durch die Versicherungsunternehmen zu begründen und festzulegen ist (vgl. Committee of European Insurance and Occupational Pensions Supervisors, 2009). Bleiben etwaige den SCR mindernde Effekte unberücksichtigt, kann dies zu einem höheren Solvenzkapitalbedarf führen, als bei einer exakten SCR-Berechnung unter Berücksichtigung möglicher Diversifikationseffekte zu erwarten wäre. Demgegenüber steht die Einsparung von Ressourcen und Kosten, die zur Bewertung der Effekte des Forward-Hedgings auf den SCR aufzubringen wären. Die Solvabilität des Versicherers ist bei Erfüllung der unter Vernachlässigung möglicher Diversifikationseffekte berechneten, im Vergleich höheren Solvenzkapitalanforderungen in jedem Fall gesichert. 


\section{Anlageentscheidung und Risikopolitik}

Die bekannte Gewinngleichung ist um den unsicheren Differenzgewinn bzw. -verlust aus dem Termingeschäft, $H\left(r_{f}-\tilde{r}_{\mathrm{A}}\right)$, zu ergänzen. Somit ist der unsichere Gewinn unter Beachtung der Bilanzgleichung (1) und der Nebenbedingungen (7) und (9) gegeben durch

$$
\tilde{\Pi}=A\left(\tilde{r}_{\mathrm{A}}+\left(\beta-r_{\mathrm{R}}\right)\left(1-c_{\alpha}\right)\right)-\frac{1}{2} \theta A^{2}+H\left(r_{f}-\tilde{r}_{\mathrm{A}}\right)
$$

Daher lautet das zu lösende Entscheidungsproblem

$$
\max _{A, H} \mathbf{E}[U(\tilde{\Pi})]
$$

Die Optimalitätsbedingungen lauten

$$
\begin{aligned}
& \frac{\mathrm{d} \mathbf{E}[U(\tilde{\Pi})]}{\mathrm{d} A}=\mathbf{E}\left[U^{\prime}\left(\tilde{\Pi}^{*}\right)\left(\tilde{r}_{\mathrm{A}}+\left(\beta-r_{\mathrm{R}}\right)\left(1-c_{\alpha}\right)-\theta A^{*}\right)\right]=0, \\
& \frac{\mathrm{d} \mathbf{E}[U(\tilde{\Pi})]}{\mathrm{d} H}=\mathbf{E}\left[U^{\prime}\left(\tilde{\Pi}^{*}\right)\left(r_{f}-\tilde{r}_{\mathrm{A}}\right)\right]=0 .
\end{aligned}
$$

Zunächst sind die Abhängigkeiten zwischen der Anlage- und der Absicherungsentscheidung zu untersuchen. Gleichsetzen der Optimalitätsbedingungen liefert unter Beachtung der Linearität des Erwartungswerts

$$
A^{*}=\frac{r_{f}-\left(r_{\mathrm{R}}-\beta\right)\left(1-c_{\alpha}\right)}{\theta} .
$$

Satz 4.1. (Separationsaussage)

Das modellierte Versicherungsunternehmen kann die Anlageentscheidung getrennt von der Absicherungsentscheidung treffen.

Satz 4.2. (Optimale Anlageentscheidung)

Das optimale Anlagevolumen ist unabhängig von dem Risikoverhalten des Versicherers. Determinanten der optimalen Anlageentscheidung sind die Grenzkosten, der Terminkurs, die aus den Rückstellungen resultierenden Zinsverpflichtungen gegenüber den Versicherungsnehmern, der Proportionalitätsfaktor zwischen Rückstellungen und Ergebnis der klassischen Versicherungstätigkeit, die Erwartungen bezüglich der unsicheren Anlagenrendite und das durch die Aufsichtsbehörde festgelegte Solvenzniveau.

Das optimale Anlagevolumen erfüllt die aufsichtsrechtlichen Regelungen von Solvency II. 
Die Integration von Terminkontrakten in das Risikomanagement des VUs führt somit zu einer deutlich vereinfachten und folglich kostensparenden Entscheidungsfindung. Das optimale Kontraktvolumen der Terminabsicherung $H^{*}$ ergibt sich aus der Optimalitätsbedingung (19). Diese kann äquivalent umgeformt werden zu

$$
\operatorname{Cov}\left[U^{\prime}\left(\tilde{\Pi}^{*}\right), \tilde{r}_{\mathrm{A}}\right]=\left(r_{f}-\mathbf{E}\left[\tilde{r}_{\mathrm{A}}\right]\right) \mathbf{E}\left[U^{\prime}\left(\tilde{\Pi}^{*}\right)\right]
$$

Umschreiben der Gewinngleichung zu

$$
\tilde{\Pi}=\tilde{r}_{\mathrm{A}}(A-H)+A\left(\beta-r_{\mathrm{R}}\right)\left(1-c_{\alpha}\right)-\frac{1}{2} \theta A^{2}+H r_{f}
$$

zeigt, dass die Risikoposition des VUs durch die Differenz aus Anlage- und Hedgingvolumen, $(A-H)$, gegeben ist.

Satz 4.3. (Optimale Risikopolitik)

Das modellierte VU legt in Abhängigkeit der Situation auf dem Terminmarkt seine optimale Hedgingentscheidung wie folgt fest:

1.Ist der Terminmarkt unverzerrt, d.h. es gilt $r_{f}=\mathbf{E}\left[\tilde{r}_{\mathrm{A}}\right]$, wählt das VU eine Vollabsicherung, $H^{*}=A^{*}$. Die optimale Wahl der Vollabsicherung gewährleistet einen sicheren Gewinn.

2.Ist der Terminmarkt durch Contango gekennzeichnet, d. h. es gilt $r_{f}>\mathbf{E}\left[\tilde{r}_{\mathrm{A}}\right]$, wählt das VU eine Überabsicherung. Es gilt $H^{*}>A^{*}$.

3.Ist der Terminmarkt durch Backwardation gekennzeichnet, d.h. es gilt $r_{f}<$ $\mathbf{E}\left[\tilde{r}_{\mathrm{A}}\right]$, wählt das VU eine Unterabsicherung. Es gilt $H^{*}<A^{*}$.

Ist der Terminmarkt verzerrt, so kann das Risiko nicht vollständig eliminiert werden. Die Terminentscheidung kann in der Bedingung

$$
\operatorname{sgn}\left(r_{f}-\mathbf{E}\left[\tilde{r}_{\mathrm{A}}\right]\right)=\operatorname{sgn}\left(H^{*}-A^{*}\right)
$$

zusammengefasst werden und ist in der Literatur als Full-Hedge-Theorem bekannt (vgl. Holthausen, 1979).

Ist der Terminmarkt durch Contango gekennzeichnet, so ist obige Differenz positiv und der Versicherer erhält eine positive Risikoprämie. Die Aktivität auf dem Terminmarkt wird mit einer positiven Risikoprämie entlohnt. Im Fall von Backwardation liegt eine ungünstige Preiskonstellation aus Sicht des Versicherers vor. Dem VU entstehen durch die Termingeschäfte aufgrund der negativen Risikoprämie zusätzliche Kosten. Somit ist eine Unterabsicherung optimal. Dies zeigt insbesondere, dass die 
angenommene perfekt negative Korrelation zwischen dem Gewinn aus der Anlagetätigkeit und dem Ergebnis des Forwardgeschäfts nicht automatisch eine Hedge-Ratio von $100 \%$ impliziert.

\section{$5 \quad$ Fazit und Ausblick}

Ziel des Beitrags war die Analyse und Bestimmung des optimalen Anlagevermögens eines VUs bei Einhaltung der europäischen Solvabilitätsvorschriften von Solvency II und Anwendung des Value at Risk. Ferner wurden die Auswirkungen eines Terminmarktzugangs auf die optimale Entscheidungspolitik hinsichtlich Anlage- und Hedgingentscheidung analysiert. Risikobehaftete Anlagen führen zu einem Endvermögensrisiko und können so die Solvenz des VUs gefährden, sodass zur Absicherung unerwartet hoher Verluste Eigenmittel in mindestens der Höhe der Solvenzkapitalanforderungen von Solvency II vorzuhalten sind. In diesem Zusammenhang wurde aufgezeigt, wie das VaR-Konzept zur Berechnung der Eigenmittelanforderungen herangezogen werden kann. Nutzt das VU keine Terminkontrakte zur Risikoabsicherung, sind die Determinanten der optimalen Anlagepolitik die Grenzkosten des Versicherers, der Grad der Risikoaversion, der Proportionalitätsfaktor zwischen Rückstellungen und Ergebnis des klassischen Versicherungsgeschäfts, die Erwartungen hinsichtlich der Anlagenrendite und das durch die Aufsichtsbehörde vorgeschriebene Solvenzniveau. Bei Anwendung des VaR-Konzepts ausgehend vom optimalen Anlagevolumen kann über die Höhe der vorzuhaltenden Eigenmittel nicht separiert entschieden werden kann. Ein simultanes Management des Aktiv- und Passivgeschäfts ist notwendig. Der Einsatz von Terminkontrakten zur Risikoabsicherung vereinfacht die Entscheidungsfindung erheblich. Die Anlageentscheidung kann getrennt von der Absicherungsentscheidung getroffen werden. Das optimale Anlagevolumen ist nun unabhängig vom Risikoverhalten des Versicherers, was ein wesentliches Merkmal der Terminabsicherung kennzeichnet. Das in dieser Arbeit aufgebaute Modell eines VUs berücksichtigt sowohl regulatorische als auch bilanzielle Anforderungen. Gemeint sind die europäischen Solvabilitätsvorschrifte, welche eine angemessene Eigenmittelausstattung des Versicherers zur Absicherung möglicher, hoher Verluste sicherstellen sollen. Das Projekt Solvency II ist noch nicht abgeschlossen und der Zeitpunkt einer europaweiten, vollumfänglichen Einführung noch nicht exakt terminiert. Gegenwärtig werden die Ergebnisse der Anfang 2013 durchgeführten Long Term Guarantees Assessment zur Untersuchung der Auswirkungen der andauernden Niedrigzinsphase auf die Versicherungsunternehmen analysiert und deren Auswirkungen auf die bestehenden Regularien diskutiert. Somit sind etwaige Änderungen und Ergänzungen der Solvency II-Richtlinie bis zur finalen zu berücksichtigen, insbesondere mit Fokus 
auf die Solvenz- und Mindestkapitalanforderungen.

\section{Literatur}

Albrecht, Peter (1995): Ansätze eines finanzwirtschaftlichen PortefeuilleManagements und ihre Bedeutung für Kapitalanlage- und Risikopolitik von Versicherungsunternehmen. Verlag Versicherungswirtschaft, Karlsruhe.

Assekurata GmbH (2013): Überschussstudie 2013: Die Überschussbeteiligung in Bedrängnis Auswirkungen politischer Zinsen auf das Vorsorgesparen.

Committee of European Insurance and Occupational Pensions Supervisors (2007): Advice to the European Commission in the Framework of the Solvency II project on Pillar I issues - further advice. Frankfurt am Main.

Committee of European Insurance and Occupational Pensions Supervisors (2009): CEIOPS' Advice for Level 2 Implementing Measures on Solvency II: SCR standard formula - Allowance of Financial Risk Mitigation Techniques. Frankfurt am Main.

Doff, René (2007): Risk Management for Insurers. Risk Books.

Farny, Dieter (2011): Versicherungsbetriebslehre. Verlag Versicherungswirtschaft, Karlsruhe.

Gesamtverband der Deutschen Versicherungswirtschaft e.V. (2013): Die Position der deutschen Versicherer 2013.

Holthausen, Duncan M. (1979): Hedging and the Competitive Firm under Price Uncertainty. The American Economic Review, Vol. 69, 989-995.

Jorion, Phillipe (2000): Value At Risk. The New Benchmark for Managing Financial Risk: The Benchmark for Controlling Market Risk. Mcgraw-Hill Professional.

Nguyen, Tristan (2008): Handbuch der wert- und risikoorientierten Steuerung von Versicherungsunternehmen. Verlag Versicherungswirtschaft, Karlsruhe.

Schradin, Heinrich R. (2003): Entwicklung der Versicherungsaufsicht. Düsseldorf.

von Neumann, John; Morgenstern, Oskar (2007): Theory of games and economic behavior. Princeton Univ. Press.

Wagner, Fred (2001): Aktuelle Fragen in der Versicherungswirtschaft. Verlag Versicherungswirtschaft, Karlsruhe.

Wiesemann, Hans-Olaf (2011): Solvency II. Wirtschaftswissenschaftliches Studium : WiSt, Heft 7 2011, S. 338-344. 